\section{Retinal Oxygen Supply and Macular Pigmentation}

There is little doubt that the macular and foveal region of the human retina is lacking in blood vessels detectable by histological means ${ }^{1}$. By subjective observation of the red corpuscles in blue light, Weale ${ }^{2}$ has recently confirmed Gescher's estimate of $1^{\circ} 30^{\prime \prime}$ for the visual angle within which the vessels of the central artery of the retina are absent. We have been unable to find any evidence that capillaries enter the foveal region from the choroidal vessels, and so it seems that in the absence of an oxygen supply directly from the blood, this region would tend to exhibit a relative anoxia. Hence, if the oxygen tension in the blood is reduced, a disturbance of central rather than peripheral vision is to be expected. In fact, experiment shows precisely the opposite effect, namely, a sparing of the central area and loss of function at the periphery under conditions of lack of oxygen 3,4 .

This unexpected result may be explained by assuming either $(a)$ that the central retinal sense cells do not have major oxygen requirements, or, $(b)$ that they are supplied by a secondary oxygen-carrying system. The first possibility is unlikely in view of the oxygen needs of the rest of the retina, and it would seem more probable that the central retina obtains its oxygen at the required tension by the intervention of a secondary process.

It is suggested that the function of the yellow macular pigment is associated with such a secondary oxygen-carrying system. Although Polyak ${ }^{5}$ has thrown some doubt on the presence of this pigment at the centre of the central fovea, the balance of evidence is in favour of pigmentation of the living foveal and macular region. The extent of retina in which the pigment is found in significant concentra. tion is roughly co-extensive with the area lacking in blood supply, and Duke-Elder ${ }^{6}$ gives instructions for its demonstration in vivo. Such a pigment, absorbing much of the incident blue light, would account well for the blue insensitivity and tritanopia of the fovea ${ }^{7,8}$.

It is curious that a yellow pigment, causing a heavy absorption of blue light, should be present in a retinal region which is, in every way, so highly developed (visual acuity, for example). Such a disadvantage might be compensated, however, if the pigment had the important function of supplying oxygen to the sense cells of the central retina.

Wald ${ }^{2}$ has recently estimated the density of the macular pigment in vivo by comparison of the peripheral and foveal photopic sensitivities. He has also extracted from human maculæ a yellow pigment which he states is a hydroxy-carotenoid or xanthophyll_-."in all probability lutein or leaf xanthophyll itself". Thus it is interesting to recall Baly's suggestion $^{10}$ that the function of carotene in green leaves is that of reducing chlorophyll-B to chlorophyll-A, the carotene being oxidized at the same time to xanthophyll. By this means the chlorophyll cycle is maintained in accordance with the following scheme :

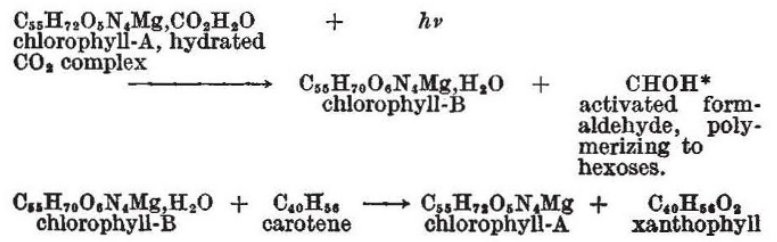

Baly's suggestion prompts the speculation that a similar xanthophyll-carotene oxidation-reaction system might exist in the macula. Xanthophyll and carotene have very similar absorption spectra, and the absorption curve of Wald's macular extract could apply as well to one as to the other. Unfortunately for this suggestion, no simple oxidation will convert carotene to xanthophyll ; nor, conversely, will any simple reduction reverse the process. It would thus appear that a xanthophyll-carotene system would scarcely be sufficiently labile by itself to serve as an oxygen-carrying mechanism for the central retina.

Although Wald's visual estimate of the macular absorption agrees approximately with the absorption spectrum of his extracts in the blue, there is, however, a discrepancy in the region of $550 \mathrm{mu}$. This discrepancy may, of course, arise from differences of receptor response within the two retinal regions (foveal and peripheral). It is significant, however, that the cytochromes have absorption maxima in this region, besides possessing maxima in the blue at similar positions to the carotenoid spectrum"1.

Since the cytochromes form a class of widely distributed biological oxidation mechanisms, and, moreover, in the reduced form, have a combined absorption similar to that of Wald's visual estimate, it is possible that these substances may be present in the macula, and, in conjunction with xanthophyll, provide the oxygen supply to this retinal region. Other mechanisms ${ }^{12}$ cannot, however, be excluded from consideration.

\section{H. J. A. Dartnall}

L. C. THOMsoN

Vision Research Unit,

Institute of Ophthalmology, Judd Street,

London.

June 30

${ }^{1}$ Duke-Elder, Sir Stewart, "Text Book of Ophthalmology" (London, Henry Kimpton).

${ }^{2}$ Weale, R. A., personal communication.

${ }^{3}$ Livingstone, P. C., Lancet, ii, 33 and 67 (1944).

${ }^{4}$ Evans, J. N., and Macfarland, R. A., Amer. J. Ophthal., 21, 968 (1938).

5 Polyak, S. L., "The Retina" (Univ. Chicago Press, 1941).

"Duke-Elder, Sir Stewart, "Text Book of Ophthalmology", p. 815.

${ }^{7}$ Willmer, E. N., and Wright, W. D., Nature, 156, 119 (1945).

8 Thomson, L. C., and Wright, W. D., J. Physiol., 105, 316 (1947).

- Wald, G., Science, 101, 635 (1945).

${ }^{10}$ Baly, E. C. C., Proc. Roy. Soc., B, 117, 218 (1935).

${ }^{11}$ Keilin, D., and Hartree, E. F., Proc. Roy. Soc., B, 127, 167 (1939). 12 Green, D. E., "Mechanisms of Biological Oxidation" (Camb. Univ. Press, 1940).

\section{Existence of L-Forms in Common Bacteria and their Possible Importance}

IN a previous communication ${ }^{1}$, we have described the morphological and cytological modifications of staphylococci after treatment with penicillin. Since then, we have investigated the effect of this anti. biotic on various bacteria : $E$. coli, $B$. anthracis, and Salmonella, Proteus, Pasteurella spp. All our observations show that penicillin reduces considerably or completely inhibits the cytoplasmic divisions, whereas the nuclear divisions take place at a normal rhythm, at least during a certain length of time. This leads to the appearance of giant forms ('large bodies') containing numerous normal or abnormal nuclei. The results are extremely clear in bacilli, and the details have been described elsewhere ${ }^{2}$.

In the course of investigations of these kind, we have been able to observe in cultures of Proteus 